\title{
C. difficile in COVID-19 Patients: Is It a Colonization Issue?
}

\author{
Levent Filik ${ }^{1}$ (1)
}

Received: 27 February 2021 / Accepted: 13 March 2021 / Published online: 12 May 2021

(C) The Author(s), under exclusive licence to Springer Science+Business Media, LLC, part of Springer Nature 2021

\section{Dear Sir,}

In their interesting article, Laszkowska et al. showed a relative increase of $C$. difficile compared to other enteropathogens in COVID-19 patients. Current knowledge show that C. difficile colonization is much more common especially in some groups (employees, students, families or previous health care facilities) regardless of COVID-19. Is there any data to establish a link to their previous community-based transient or permanent colonization? It is also much better if a previous antibiotic use would be detailed to clarify the role of C.difficile as a significant contributor to diarrhea in COVID-19 positive patients.

\section{Reply}

We thank Dr. Filik for the question as to whether the Clostridioides difficile detected represented colonization or infection [1]. Testing at our institution is via PCR for the toxin B gene of $C$. difficile [2]. To decrease false positive results, tests are only run on unformed stool samples, and there is an institutional electronic "hard stop" that prohibits stool PCR testing in patients who have received laxatives within the previous $24 \mathrm{~h}$. These restrictions may help ensure that only patients with clinically significant diarrhea are tested, though the test itself does not distinguish carriers from those who are infected.

The purpose of the study was to examine how COVID-19 impacted $C$. difficile infection and other enteric infections. Gastrointestinal symptoms including diarrhea are present in up to one third of patients with COVID-19, yet we found that rates of $C$. difficile infection were similar among those with COVID-19 compared with those without it (5.1\%

\section{Levent Filik}

leventfilik@yahoo.co.uk

1 Gastroenterology Department, Health Sciences University, Ankara Research Hospital, Ankara, Turkey versus $8.2 \%$, respectively, $p=0.33$ ) $[1,3,4]$. Therefore, the main study finding - that $C$. difficile infection rates are similar regardless of COVID-19 infection status-is likely to hold true even if some colonized patients were classified as infected.

Monika Laszkowska MD MS ${ }^{\mathrm{a}}$

Judith Kim MD ${ }^{\mathrm{b}}$

Chin Hur MD MPH ${ }^{\mathrm{b}, \mathrm{c}, \mathrm{d}}$

Daniel E. Freedberg MD MS

${ }^{a}$ Department of Subspecialty Medicine, Gastroenterology, Hepatology, and Nutrition Service, Memorial Sloan Kettering Cancer Center, New York, NY

${ }^{\mathrm{b}}$ Department of Medicine, Division of Digestive and Liver Diseases, Columbia University Irving Medical Center, New York, NY

${ }^{\mathrm{c}}$ Department of Medicine, Division of General Medicine, Columbia University Irving Medical Center, New York, NY

${ }^{\mathrm{d}}$ Herbert Irving Comprehensive Cancer Center, Columbia University Irving Medical Center, New York, NY

Correspondence:

Monika Laszkowska, LaszkowM@mskcc.org.

\section{References}

1. Laszkowska M, Kim J, Faye AS et al. Prevalence of clostridioides difficile and other gastrointestinal pathogens in patients with COVID19. Dig Dis Sci. (Epub ahead of print). https://doi.org/10.1007/ s10620-020-06760-y.

2. Polage CR, Gyorke CE, Kennedy MA et al. Overdiagnosis of clostridium difficile infection in the molecular test era. JAMA Intern Med 2015;175:1792-1801.

3. Nobel YR, Phipps M, Zucker J et al. Gastrointestinal symptoms and coronavirus disease 2019: a case-control study from the United States. Gastroenterology 2020;159:373-375.

4. Laszkowska M, Faye AS, Judith, et al. Disease course and outcomes of COVID-19 among hospitalized patients with gastrointestinal manifestations. Clin Gastroenterol Hepatol. 2020.https://doi.org/10.1016/j. cgh.2020.09.037 (Epub ahead of print. PMID: 33007514).

Publisher's Note Springer Nature remains neutral with regard to jurisdictional claims in published maps and institutional affiliations. 